\title{
Ramanujan's formulas for the explicit evaluation of the Rogers-Ramanujan continued fraction and theta-functions
}

\author{
by
}

SoON-Yi KANG (Urbana, IL)

1. Introduction. Let, for $|q|<1$,

$$
R(q):=\frac{q^{1 / 5}}{1}+\frac{q}{1}+\frac{q^{2}}{1}+\frac{q^{3}}{1}+\cdots
$$

and

$$
S(q):=-R(-q)
$$

denote the Rogers-Ramanujan continued fractions. This famous continued fraction was introduced by L. J. Rogers [19] in 1894 and rediscovered by S. Ramanujan in approximately 1912. In his first two letters to G. H. Hardy [16], Ramanujan communicated several results concerning $R(q)$. In particular, he asserted that

$$
\begin{aligned}
R\left(e^{-2 \pi}\right) & =\sqrt{\frac{5+\sqrt{5}}{2}}-\frac{\sqrt{5}+1}{2}, \\
S\left(e^{-\pi}\right) & =\sqrt{\frac{5-\sqrt{5}}{2}}-\frac{\sqrt{5}-1}{2},
\end{aligned}
$$

and

$$
R\left(e^{-2 \pi \sqrt{5}}\right)=\frac{\sqrt{5}}{1+\left(5^{3 / 4}\left(\frac{\sqrt{5}-1}{2}\right)^{5 / 2}-1\right)^{1 / 5}}-\frac{\sqrt{5}+1}{2} .
$$

These identities were first proved by G. N. Watson [20], [21], and the latter two identities were also established by K. G. Ramanathan [10]. Four more evaluations of $R\left(e^{-2 \pi \sqrt{n}}\right)$ when $n=4,9,16$, and 64 are found on p. 311 of Ramanujan's first notebook [15]. Ramanathan [11] gave a proof of the evaluation of $R\left(e^{-4 \pi}\right)$, but proofs for the other three were first given by B. C. Berndt and H. H. Chan [6]. Several further evaluations of $R(q)$ and

1991 Mathematics Subject Classification: Primary 33D10; Secondary 11F27, 40A15. 
$S(q)$ were recorded by Ramanujan on pages 46 and 210 in his lost notebook [18]. These evaluations have been proved by Ramanathan [12]-[14], Berndt and Chan [6], and Berndt, Chan, and L.-C. Zhang [7].

It is noticeable that the evaluations of $R\left(e^{-2 \pi \sqrt{n}}\right)$ and $S\left(e^{-\pi \sqrt{n}}\right)$ for several rational numbers $n$ were first made in a uniform way by Ramanathan [13] by using Kronecker's limit formula. However, Ramanathan's method could not possibly have been known to Ramanujan. Furthermore, his method cannot be applied to all the evaluations of $R(q)$ stated by Ramanujan.

Berndt, Chan, and Zhang [7] recently derived the first formulas for the explicit evaluations of $R\left(e^{-2 \pi \sqrt{n}}\right)$ and $S\left(e^{-\pi \sqrt{n}}\right)$ for positive rational numbers $n$ in terms of Ramanujan-Weber class invariants by employing two beautiful theorems of $R(q)$ given in (1.7) and (1.8) below. To describe these two theorems, we need to define Ramanujan's function $f(-q)$ by

$$
f(-q):=(q ; q)_{\infty}=: q^{-1 / 24} \eta(z), \quad q=e^{2 \pi i z}, \quad \operatorname{Im} z>0,
$$

where $\eta(z)$ denotes the Dedekind eta-function and we employ the customary notation

$$
(a ; q)_{\infty}:=\prod_{n=0}^{\infty}\left(1-a q^{n}\right), \quad|q|<1 .
$$

Two of the most important formulas about $R(q)$ are given by

$$
\frac{1}{R(q)}-1-R(q)=\frac{f\left(-q^{1 / 5}\right)}{q^{1 / 5} f\left(-q^{5}\right)}
$$

and

$$
\frac{1}{R^{5}(q)}-11-R^{5}(q)=\frac{f^{6}(-q)}{q f^{6}\left(-q^{5}\right)} .
$$

The equalities in (1.7) and (1.8) were found by Watson in Ramanujan's notebooks and proved by him [20].

To evaluate $R(q)$ explicitly for a certain value of $q$, it is clear that it suffices to evaluate the right side of either (1.7) or (1.8). On page 46 in his lost notebook [18], Ramanujan offers some identities which lead to the evaluation of the right side of (1.8). In Section 2, we prove these identities and also derive analogous identities leading to the evaluation of the right side of (1.7). Berndt, Chan, and Zhang [7] have also established formulas leading to the evaluations of the right sides of (1.7) and (1.8). We show that Ramanujan's formulas and our formulas also lead to the formulas of Berndt, Chan, and Zhang, which actually are more amenable to specific calculations than those of Ramanujan. At the time of the writing of their paper [7], Berndt, Chan, and Zhang were unaware of this material in Ramanujan's lost notebook. 
The theorems in Section 2 are the main formulas of this paper, from which we can derive two formulas for the Rogers-Ramanujan continued fraction found on page 208 in Ramanujan's lost notebook. They are the first known formulas for computing directly the values of the Rogers-Ramanujan continued fraction. The proofs of these formulas are given in Section 3.

In Section 4, we illustrate our main theorems and the formulas proved in Section 3 by calculating some values of $R(q)$.

In Section 5, we briefly discuss evaluation of the theta-functions $\varphi(q)$ and $\psi(q)$ defined by

$$
\varphi(q):=\sum_{n=-\infty}^{\infty} q^{n^{2}}=\frac{(-q ;-q)_{\infty}}{(q ;-q)_{\infty}}
$$

and

$$
\psi(q):=\sum_{n=0}^{\infty} q^{n(n+1) / 2}=\frac{\left(q^{2} ; q^{2}\right)_{\infty}}{\left(q ; q^{2}\right)_{\infty}}
$$

where the infinite product representations arise from Jacobi's triple product identity [2, p. 35, Entry 19]. For certain values of $q$, our main theorems contain representations of these theta-functions as well as the aforementioned eta-functions in terms of class invariants defined by (1.12) and (1.13) below.

In the final section, we provide tables summarizing the values of $R(q)$ and $S(q)$ that appear in the literature.

We complete this introduction with a few basic facts about class invariants. Define, for $|q|<1$,

$$
\chi(-q):=\left(q ; q^{2}\right)_{\infty} .
$$

The class invariants are defined by

$$
G_{n}:=2^{-1 / 4} q^{-1 / 24} \chi(q)
$$

and

$$
g_{n}:=2^{-1 / 4} q^{-1 / 24} \chi(-q),
$$

where $q=\exp (-\pi \sqrt{n})$.

As usual, in the theory of elliptic functions, let $k:=k(q), 0<k<1$, denote the modulus. The singular modulus $k_{n}$ is defined by $k_{n}:=k\left(e^{-\pi \sqrt{n}}\right)$, where $n$ is a natural number. Following Ramanujan, set $\alpha=k^{2}$ and $\alpha_{n}=k_{n}^{2}$. Since $\chi(q)=2^{1 / 6}\{\alpha(1-\alpha) / q\}^{-1 / 24}$ and $\chi(-q)=2^{1 / 6}(1-\alpha)^{1 / 12}(\alpha / q)^{-1 / 24}$ [2, p. 124], it follows from (1.12) and (1.13) that

$$
G_{n}=\left\{4 \alpha_{n}\left(1-\alpha_{n}\right)\right\}^{-1 / 24}
$$

and

$$
g_{n}=2^{-1 / 12}\left(1-\alpha_{n}\right)^{1 / 12} \alpha_{n}^{-1 / 24} .
$$




\section{Main formulas}

Theorem 2.1 ([18], p. 46). Let

$$
t_{1}:=t_{1}(q):=q^{1 / 6} \frac{\chi(-q)}{\chi\left(-q^{5}\right)} \quad \text { and } \quad s_{1}:=s_{1}(q):=\frac{\varphi(-q)}{\varphi\left(-q^{5}\right)} .
$$

Then

(i) $\frac{f(-q)}{q^{1 / 6} f\left(-q^{5}\right)}=\frac{s_{1}}{t_{1}} ; \quad$ (ii) $\frac{f\left(-q^{2}\right)}{q^{1 / 3} f\left(-q^{10}\right)}=\frac{s_{1}}{t_{1}^{2}} ; \quad$ (iii) $\quad \frac{\psi(q)}{\sqrt{q} \psi\left(q^{5}\right)}=\frac{s_{1}}{t_{1}^{3}}$;

(iv) $s_{1}^{2}=\frac{1}{2}\left(\left(1+t_{1}^{6}\right)+\sqrt{\left(1+t_{1}^{6}\right)^{2}-20 t_{1}^{6}}\right)$.

REMARK. Instead of (iv), Ramanujan actually stated

$$
s_{1}=\frac{1}{2}\left(\sqrt{1+2 \sqrt{5} t_{1}^{3}+t_{1}^{6}}+\sqrt{1-2 \sqrt{5} t_{1}^{3}+t_{1}^{6}}\right)
$$

with a slight misprint. But in applications, it is more convenient to use the equality in (iv) instead of (2.1) because of the nature of $t_{1}$.

Proof (of Theorem 2.1). (i) Set $t=t_{1}$ and $s=s_{1}$ throughout the proof. By (1.11), we have

$$
t=q^{1 / 6} \frac{\left(q ; q^{2}\right)_{\infty}}{\left(q^{5} ; q^{10}\right)_{\infty}} .
$$

Using the definition of $f(-q)$ in (1.6), Euler's identity,

$$
(-q ; q)_{\infty}=\frac{1}{\left(q ; q^{2}\right)_{\infty}},
$$

(1.9), and (2.2), we deduce that

$$
\begin{aligned}
\frac{f(-q)}{q^{1 / 6} f\left(-q^{5}\right)} & =\frac{(q ; q)_{\infty}}{q^{1 / 6}\left(q^{5} ; q^{5}\right)_{\infty}}=\frac{(q ; q)_{\infty}}{(-q ; q)_{\infty}} \cdot \frac{\left(-q^{5} ; q^{5}\right)_{\infty}}{\left(q^{5} ; q^{5}\right)_{\infty}} \cdot \frac{\left(q^{5} ; q^{10}\right)_{\infty}}{q^{1 / 6}\left(q ; q^{2}\right)_{\infty}} \\
& =\frac{\varphi(-q)}{\varphi\left(-q^{5}\right)} \cdot \frac{\chi\left(-q^{5}\right)}{q^{1 / 6} \chi(-q)}=\frac{s}{t} .
\end{aligned}
$$

(ii) Using in turn (1.6), Euler's identity (2.3), (1.9), and (2.2), we find that

$$
\begin{aligned}
\frac{f\left(-q^{2}\right)}{q^{1 / 3} f\left(-q^{10}\right)} & =\frac{\left(q^{2} ; q^{2}\right)_{\infty}}{q^{1 / 3}\left(q^{10} ; q^{10}\right)_{\infty}} \\
& =\frac{(q ; q)_{\infty}}{(-q ; q)_{\infty}} \cdot \frac{\left(-q^{5} ; q^{5}\right)_{\infty}}{\left(q^{5} ; q^{5}\right)_{\infty}} \cdot \frac{\left(q^{5} ; q^{5}\right)_{\infty}\left(q^{5} ; q^{10}\right)_{\infty}}{(q ; q)_{\infty}\left(q ; q^{2}\right)_{\infty}} \cdot \frac{\left(q^{2} ; q^{2}\right)_{\infty}}{q^{1 / 3}\left(q^{10} ; q^{10}\right)_{\infty}} \\
& =\frac{\varphi(-q)}{\varphi\left(-q^{5}\right)} \cdot \frac{\left(q^{5} ; q^{10}\right)_{\infty}^{2}}{q^{1 / 3}\left(q ; q^{2}\right)_{\infty}^{2}}=\frac{s}{t^{2}}
\end{aligned}
$$


(iii) Applying (1.10), Euler's identity (2.3), (1.9), and (2.2), we have

$$
\begin{aligned}
\frac{\psi(q)}{q^{1 / 2} \psi\left(q^{5}\right)}= & \frac{\left(q^{2} ; q^{2}\right)_{\infty}}{q^{1 / 2}\left(q ; q^{2}\right)_{\infty}} \cdot \frac{\left(q^{5} ; q^{10}\right)_{\infty}}{\left(q^{10} ; q^{10}\right)_{\infty}} \\
= & \frac{(q ; q)_{\infty}}{(-q ; q)_{\infty}} \cdot \frac{\left(-q^{5} ; q^{5}\right)_{\infty}}{\left(q^{5} ; q^{5}\right)_{\infty}} \cdot \frac{\left(q^{5} ; q^{5}\right)_{\infty}\left(q^{5} ; q^{10}\right)_{\infty}}{(q ; q)_{\infty}\left(q ; q^{2}\right)_{\infty}} \\
& \times \frac{\left(q^{2} ; q^{2}\right)_{\infty}}{q^{1 / 2}\left(q ; q^{2}\right)_{\infty}} \cdot \frac{\left(q^{5} ; q^{10}\right)_{\infty}}{\left(q^{10} ; q^{10}\right)_{\infty}} \\
= & \frac{\varphi(-q)}{\varphi\left(-q^{5}\right)} \cdot \frac{\left(q^{5} ; q^{10}\right)_{\infty}^{3}}{q^{1 / 2}\left(q ; q^{2}\right)_{\infty}^{3}}=\frac{s}{t^{3}}
\end{aligned}
$$

(iv) In the sequel, set

$$
P_{1}:=P_{1}(q):=\frac{f(-q)}{q^{1 / 6} f\left(-q^{5}\right)} \quad \text { and } \quad Q_{1}:=\frac{f\left(-q^{2}\right)}{q^{1 / 3} f\left(-q^{10}\right)} .
$$

Recall that [3, p. 206, Entry 53]

$$
P_{1} Q_{1}+\frac{5}{P_{1} Q_{1}}=\left(\frac{P_{1}}{Q_{1}}\right)^{3}+\left(\frac{Q_{1}}{P_{1}}\right)^{3} .
$$

Since $P_{1}=s / t$ and $Q_{1}=s / t^{2}$ by (i) and (ii), respectively, the equation above can be simplified to

$$
s^{4}-\left(1+t^{6}\right) s^{2}+5 t^{6}=0 .
$$

Thus (iv) follows immediately from the equation above by using the quadratic formula.

Proposition 2.2. If $P_{1}$ and $Q_{1}$ are defined as in (2.4), then

(i) if $2 c=Q_{1}^{6}+11$, then $R^{5}\left(q^{2}\right)=\sqrt{c^{2}+1}-c$,

(ii) if $2 c=P_{1}^{6}(-q)+11$, then $S^{5}(q)=\sqrt{c^{2}+1}+c$.

Pro of. Solve the quadratic equations

$$
\frac{1}{R^{5}\left(q^{2}\right)}-11-R^{5}\left(q^{2}\right)=\frac{f^{6}\left(-q^{2}\right)}{q^{2} f^{6}\left(-q^{10}\right)}
$$

and

$$
\frac{1}{S^{5}(q)}+11-S^{5}(q)=\frac{f^{6}(q)}{q f^{6}\left(q^{5}\right)} .
$$

Equations (2.5) and (2.6) are obtained from (1.8) with $q$ replaced by $q^{2}$ and $-q$, respectively.

Theorem 2.3 below is analogous to Theorem 2.1, but it was not stated anywhere by Ramanujan. 
Theorem 2.3. Let

$$
t_{2}:=t_{2}(q):=q^{1 / 5} \frac{\chi\left(-q^{1 / 5}\right)}{\chi\left(-q^{5}\right)} \quad \text { and } \quad s_{2}:=s_{2}(q):=\frac{\varphi\left(-q^{1 / 5}\right)}{\varphi\left(-q^{5}\right)} .
$$

Then

(i) $\frac{f\left(-q^{1 / 5}\right)}{q^{1 / 5} f\left(-q^{5}\right)}=\frac{s_{2}}{t_{2}} ; \quad$ (ii) $\quad \frac{f\left(-q^{2 / 5}\right)}{q^{2 / 5} f\left(-q^{10}\right)}=\frac{s_{2}}{t_{2}^{2}} ; \quad$ (iii) $\quad \frac{\psi\left(q^{1 / 5}\right)}{q^{3 / 5} \psi\left(q^{5}\right)}=\frac{s_{2}}{t_{2}^{3}}$;

(iv) $s_{2}=\frac{1-2 t_{2}-2 t_{2}^{2}+t_{2}^{3}+\sqrt{1-4 t_{2}-10 t_{2}^{3}-4 t_{2}^{5}+t_{2}^{6}}}{2}$.

Proof. Set $t=t_{2}$ and $s=s_{2}$ in the proof. From (1.11), we find that

$$
t=q^{1 / 5} \frac{\left(q^{1 / 5} ; q^{2 / 5}\right)_{\infty}}{\left(q^{5} ; q^{10}\right)_{\infty}} .
$$

The proofs of (i)-(iii) are similar to those of Theorem 2.1(i)-(iii), respectively.

To prove (iv), set

$$
P_{2}:=P_{2}(q)=\frac{f\left(-q^{1 / 5}\right)}{q^{1 / 5} f\left(-q^{5}\right)} \quad \text { and } \quad Q_{2}:=\frac{f\left(-q^{2 / 5}\right)}{q^{2 / 5} f\left(-q^{10}\right)} .
$$

Then by Entry 58 in [3, p. 212], we have

$$
P_{2} Q_{2}+\frac{25}{P_{2} Q_{2}}=\left(\frac{Q_{2}}{P_{2}}\right)^{3}-4\left(\frac{Q_{2}}{P_{2}}\right)^{2}-4\left(\frac{P_{2}}{Q_{2}}\right)^{2}+\left(\frac{P_{2}}{Q_{2}}\right)^{3} .
$$

Since $P_{2}=s / t$ and $Q_{2}=s / t^{2}$ from (i) and (ii), respectively, we derive that

$$
\frac{s^{2}}{t^{3}}+25 \frac{t^{3}}{s^{2}}=\frac{1}{t^{3}}-4 \frac{1}{t^{2}}-4 t^{2}+t^{3} \text {. }
$$

Multiply both sides of (2.10) by $s^{2} t^{3}$ to deduce

$$
s^{4}-\left(1-4 t-4 t^{5}+t^{6}\right) s^{2}+25 t^{6}=0 .
$$

Solutions of this equation for $s$ are

or

$$
s=\frac{1-2 t-2 t^{2}+t^{3} \pm \sqrt{1-4 t-10 t^{3}-4 t^{5}+t^{6}}}{2},
$$

$$
s=\frac{-1+2 t+2 t^{2}-t^{3} \pm \sqrt{1-4 t-10 t^{3}-4 t^{5}+t^{6}}}{2} .
$$

But since $t$ and $s$ approach 0 and 1, respectively, as $q$ approaches 0 , the appropriate solution for $s$ is

$$
s=\frac{1-2 t-2 t^{2}+t^{3}+\sqrt{1-4 t-10 t^{3}-4 t^{5}+t^{6}}}{2} .
$$


Proposition 2.4. If $P_{2}$ and $Q_{2}$ are defined as in (2.8), then

(i) if $2 c=Q_{2}+1$, then $R\left(q^{2}\right)=\sqrt{c^{2}+1}-c$,

(ii) if $2 c=P_{2}(-q)+1$, then $S(q)=\sqrt{c^{2}+1}+c$.

Proof. Solve the quadratic equations

$$
\frac{1}{R\left(q^{2}\right)}-1-R\left(q^{2}\right)=\frac{f\left(-q^{2 / 5}\right)}{q^{2 / 5} f\left(-q^{10}\right)}
$$

and

$$
\frac{1}{S(q)}+1-S(q)=\frac{f\left(q^{1 / 5}\right)}{q^{1 / 5} f\left(q^{5}\right)} .
$$

Equations (2.12) and (2.13) are derived from (1.7) with $q$ replaced by $q^{2}$ and $-q$, respectively.

Let $q=\exp (-\pi \sqrt{n})$ for a positive rational number $n$. Recall that $t_{1}(q)$ and $t_{2}(q)$ are defined in Theorems 2.1 and 2.3, respectively. Then by (1.12) and (1.13),

$$
\begin{aligned}
t_{1}^{6}(q) & =\left(\frac{g_{n}}{g_{25 n}}\right)^{6}, & t_{1}^{6}(-q) & =-\left(\frac{G_{n}}{G_{25 n}}\right)^{6}, \\
t_{2}(q) & =\frac{g_{n / 25}}{g_{25 n}}, & t_{2}(-q) & =-\frac{G_{n / 25}}{G_{25 n}} .
\end{aligned}
$$

In [7], utilizing some theta-function identities and modular equations of degree 5, Berndt, Chan, and Zhang found relations among $P_{1}, Q_{1}, t_{1}(q)$, and $t_{1}(-q)$, where $P_{1}$ and $Q_{1}$ are defined by (2.4), and by using modular equations of degree 25, they also discovered relations among $P_{2}, Q_{2}, t_{2}$, and $t_{2}(-q)$, where $P_{2}$ and $Q_{2}$ are defined by (2.8). All the relations they found in [7] are actually equivalent to parts of Theorems 2.1 and 2.3, if $q$ is confined to be $\exp (-\pi \sqrt{n})$. In particular, they showed

$$
\frac{Q_{2}}{\sqrt{5} T}+\frac{\sqrt{5} T}{Q_{2}}=\left(T+T^{-1}\right)^{2}\left(\frac{T+T^{-1}}{\sqrt{5}}-\frac{\sqrt{5}}{T+T^{-1}}\right),
$$

where

From Theorem 2.3(ii), we have

$$
T:=\sqrt{\frac{g_{25 n}}{g_{n / 25}}}=t_{2}^{-1 / 2} .
$$

$$
s_{2}=Q_{2} t_{2}^{2}
$$

And recall from (2.11) that

$$
s_{2}^{4}-\left(1-4 t_{2}-4 t_{2}^{5}+t_{2}^{6}\right) s_{2}^{2}+25 t_{2}^{6}=0 .
$$

Substituting (2.17) into the equality above, we deduce that

$$
t_{2}^{8} Q_{2}^{4}+25 t_{2}^{6}=t_{2}^{4} Q_{2}^{2}-4 t_{2}^{5} Q_{2}^{2}-4 t_{2}^{9} Q_{2}^{2}+t_{2}^{10} Q_{2}^{2} .
$$


Since $t_{2}=T^{-2}$, after multiplying both sides by $T^{16} Q_{2}^{-2}$, we obtain

$$
Q_{2}^{2}+25 T^{4} Q_{2}^{-2}=T^{8}-4 T^{6}-4 T^{-2}+T^{-4} .
$$

Adding $10 T^{2}$ to both sides of (2.18) yields

$$
\left(Q_{2}+5 T^{2} Q_{2}^{-1}\right)^{2}=\left(T^{4}-2 T^{2}+T^{-2}-2\right)^{2},
$$

so we have

$$
Q_{2}+5 T^{2} Q_{2}^{-1}=T^{4}-2 T^{2}+T^{-2}-2,
$$

because the square roots of both sides of (2.19) have leading terms $q^{-2 / 5}$. By dividing both sides of $(2.20)$ by $\sqrt{5 T}$, we deduce (2.16). We can derive all the other formulas discussed in [7] from our main theorems by the same method.

\section{Ramanujan's explicit formulas for the Rogers-Ramanujan} continued fraction

Theorem 3.1 ([18], p. 208). Let $t_{2}$ be given in Theorem 2.3. Then

$$
\begin{aligned}
R(q)=\frac{1}{4 t_{2}} & \left(\left(1+t_{2} \frac{\sqrt{5}+1}{2}\right) \sqrt{1-t_{2}}\right. \\
& \left.-\sqrt{\left(1-t_{2}\right)\left(1+t_{2} \frac{\sqrt{5}+1}{2}\right)^{2}-2 t_{2}(\sqrt{5}+1)}\right) \\
\times & -\left(1-t_{2} \frac{\sqrt{5}-1}{2}\right) \sqrt{1-t_{2}} \\
& \left.+\sqrt{\left(1-t_{2}\right)\left(1-t_{2} \frac{\sqrt{5}-1}{2}\right)^{2}+2 t_{2}(\sqrt{5}-1)}\right),
\end{aligned}
$$

(ii) $\quad R\left(q^{2}\right)=\frac{1}{4 t_{2}^{2}}\left(\left(1-t_{2} \frac{\sqrt{5}+1}{2}\right) \sqrt{1-t_{2}}\right.$

$$
\begin{gathered}
\left.-\sqrt{\left(1-t_{2}\right)\left(1+t_{2} \frac{\sqrt{5}+1}{2}\right)^{2}-2 t_{2}(\sqrt{5}+1)}\right) \\
\times\left(-\left(1+t_{2} \frac{\sqrt{5}-1}{2}\right) \sqrt{1-t_{2}}\right. \\
\left.+\sqrt{\left(1-t_{2}\right)\left(1-t_{2} \frac{\sqrt{5}-1}{2}\right)^{2}+2 t_{2}(\sqrt{5}-1)}\right) .
\end{gathered}
$$

Proof. (i) Set $t=t_{2}$ throughout the proof. From (1.7) and Theorem 2.3(i), (iv), we have

$$
\frac{1}{R(q)}-1-R(q)=\frac{1-2 t-2 t^{2}+t^{3}+\sqrt{1-4 t-10 t^{3}-4 t^{5}+t^{6}}}{2 t}
$$


which is equivalent to

$$
\frac{1}{R(q)}-R(q)=\frac{1-2 t^{2}+t^{3}+\sqrt{1-4 t-10 t^{3}-4 t^{5}+t^{6}}}{2 t} .
$$

Motivated by the fact that $R(q)$ are units when $q=e^{-\pi \sqrt{n}}$ (see [7]), let us assume that $R(q)$ can be written a product of two units such as

$$
R(q)=(\sqrt{a+1}-\sqrt{a})(\sqrt{b}-\sqrt{b-1}) .
$$

Then

$$
\frac{1}{R(q)}-R(q)=2(\sqrt{a b}+\sqrt{(a+1)(b-1)}) .
$$

From (3.2) and (3.4), we may set

$$
\sqrt{a b}=\sqrt{\frac{\left(1-2 t^{2}+t^{3}\right)^{2}}{16 t^{2}}}
$$

and

$$
\sqrt{(a+1)(b-1)}=\sqrt{\frac{1-4 t-10 t^{3}-4 t^{5}+t^{6}}{16 t^{2}}} .
$$

Equating (3.5) and (3.6) yields

$$
a=\left(\frac{\sqrt{5}+1}{2}\right)\left(\frac{1-t}{4 t}\right)\left(1-t \frac{\sqrt{5}-1}{2}\right)^{2}
$$

and

$$
b=\left(\frac{\sqrt{5}-1}{2}\right)\left(\frac{1-t}{4 t}\right)\left(1+t \frac{\sqrt{5}+1}{2}\right)^{2} .
$$

Hence (i) follows from (3.3), (3.7), and (3.8).

(ii) The proof of the formula for $R\left(q^{2}\right)$ is similar to that of $R(q)$. By (1.7) and Theorem 2.3(ii), (iv),

$$
\frac{1}{R\left(q^{2}\right)}-R\left(q^{2}\right)=\frac{1-2 t+t^{3}+\sqrt{1-4 t-10 t^{3}-4 t^{5}+t^{6}}}{2 t^{2}} .
$$

As before, let

$$
R\left(q^{2}\right)=(\sqrt{a+1}-\sqrt{a})(\sqrt{b}-\sqrt{b-1}) .
$$

Then

$$
\frac{1}{R\left(q^{2}\right)}-R\left(q^{2}\right)=2(\sqrt{a b}+\sqrt{(a+1)(b-1)}) .
$$

Equating

$$
\sqrt{a b}=\sqrt{\frac{\left(1-2 t+t^{3}\right)^{2}}{16 t^{4}}}
$$


and

we deduce that

$$
\sqrt{(a+1)(b-1)}=\sqrt{\frac{1-4 t-10 t^{3}-4 t^{5}+t^{6}}{16 t^{4}}}
$$

$$
a=\left(\frac{\sqrt{5}+1}{2}\right)\left(\frac{1-t}{4 t^{2}}\right)\left(1+t \frac{\sqrt{5}-1}{2}\right)^{2}
$$

and

$$
b=\left(\frac{\sqrt{5}-1}{2}\right)\left(\frac{1-t}{4 t^{2}}\right)\left(1-t \frac{\sqrt{5}+1}{2}\right)^{2} .
$$

We complete the proof by utilizing (3.12) and (3.13) in (3.10).

Theorem 3.2 ([18], p. 208). Let $t_{1}$ be given in Theorem 2.1 and let

$$
k:=R(q) R^{2}\left(q^{2}\right) .
$$

Then

$$
R(q)=k^{1 / 5}\left(\frac{1-k}{1+k}\right)^{2 / 5} \quad \text { and } \quad R\left(q^{2}\right)=k^{2 / 5}\left(\frac{1+k}{1-k}\right)^{1 / 5} .
$$

Furthermore,

$$
\begin{aligned}
k= & \frac{1}{4 t_{1}^{6}}\left(\sqrt{1-t_{1}^{6}}-\sqrt{1-t_{1}^{6}\left(\frac{\sqrt{5}+1}{2}\right)^{6}}\right) \\
& \times\left(\sqrt{1-t_{1}^{6}\left(\frac{\sqrt{5}-1}{2}\right)^{6}}-\sqrt{1-t_{1}^{6}}\right)
\end{aligned}
$$

and

(iii)

$$
\begin{aligned}
\frac{1-k}{1+k}= & \frac{1}{4}\left(\sqrt{\left(\frac{\sqrt{5}+1}{2}\right)^{6}-t_{1}^{6}}-\sqrt{1-t_{1}^{6}}\right) \\
& \times\left(\sqrt{\left(\frac{\sqrt{5}-1}{2}\right)^{6}-t_{1}^{6}}+\sqrt{1-t_{1}^{6}}\right) .
\end{aligned}
$$

Pr o of. Set $t=t_{1}$ in the proof. Equalities (i) were found by Ramanujan [15, p. 362], and first proved by Andrews, Berndt, Jacobsen, and Lamphere [1, Entry 24]. So it suffices to prove (ii) and (iii).

Utilizing (i) in (1.8), we see that

$$
\begin{aligned}
\frac{f^{6}(-q)}{q f^{6}\left(-q^{5}\right)} & =\frac{1}{k}\left(\frac{1+k}{1-k}\right)^{2}-11-k\left(\frac{1-k}{1+k}\right)^{2} \\
& =\left(\frac{1+k-k^{2}}{k}\right)\left(\frac{1-4 k-k^{2}}{1-k^{2}}\right)^{2} .
\end{aligned}
$$


Hence, by Theorem 2.1(i),

or

$$
\frac{s_{1}^{6}}{t_{1}^{6}}=\left(\frac{1+k-k^{2}}{k}\right)\left(\frac{1-4 k-k^{2}}{1-k^{2}}\right)^{2},
$$

But, since

$$
t^{6}=\left(\frac{k}{1+k-k^{2}}\right)\left(\frac{1-k^{2}}{1-4 k-k^{2}}\right)^{2}\left(\frac{\varphi(-q)}{\varphi\left(-q^{5}\right)}\right)^{6} .
$$

$$
\frac{\varphi^{2}(-q)}{\varphi^{2}\left(-q^{5}\right)}=\frac{1-4 k-k^{2}}{1-k^{2}}
$$

as proved in [9, Theorem 4.2], we obtain

$$
t^{6}=\frac{k\left(1-4 k-k^{2}\right)}{\left(1-k^{2}\right)\left(1+k-k^{2}\right)} .
$$

Rearranging (3.14), we find that

$$
t^{6} k^{4}+\left(1-t^{6}\right) k^{3}+\left(4-2 t^{6}\right) k^{2}-\left(1-t^{6}\right) k+t^{6}=0,
$$

which can be expressed as

$$
t^{6}\left(\frac{1}{k}-k\right)^{2}-\left(1-t^{6}\right)\left(\frac{1}{k}-k\right)+4=0 .
$$

By the quadratic formula,

$$
\frac{1}{k}-k=2\left(\frac{\left(1-t^{6}\right)+\sqrt{t^{12}-18 t^{6}+1}}{4 t^{6}}\right) .
$$

As in the proof of Theorem 3.1, let

$$
k=(\sqrt{a+1}-\sqrt{a})(\sqrt{b}-\sqrt{b-1}) .
$$

Then

$$
\frac{1}{k}-k=2(\sqrt{a b}+\sqrt{(a+1)(b-1)}) .
$$

Comparing this with (3.16), we may set

$$
\sqrt{a b}=\sqrt{\frac{\left(1-t^{6}\right)^{2}}{16 t^{12}}} \text { and } \sqrt{(a+1)(b-1)}=\sqrt{\frac{t^{12}-18 t^{6}+1}{16 t^{12}}} .
$$

Hence we can conclude that

$$
a=\left(\frac{1+\sqrt{5}}{2}\right)^{3} \frac{1-t^{6}}{4 t^{6}}
$$

and

$$
b=\left(\frac{\sqrt{5}-1}{2}\right)^{3} \frac{1-t^{6}}{4 t^{6}} .
$$

The formula (ii) follows from (3.17)-(3.19). 
We prove (iii) in a similar way. Let $u=(1-k) /(1+k)$. Substituting $k=(1-u) /(1+u)$ in $(3.15)$, we find that

$$
4 t^{6}-\left(1-t^{6}\right)\left(\frac{1}{u}-u\right)+\left(\frac{1}{u}-u\right)^{2}=0,
$$

and hence, by the quadratic formula,

$$
\frac{1}{u}-u=2\left(\frac{\left(1-t^{6}\right)+\sqrt{t^{12}-18 t^{6}+1}}{4}\right) .
$$

As, if

$$
u=(\sqrt{a+1}-\sqrt{a})(\sqrt{b}-\sqrt{b-1}),
$$

then

$$
\frac{1}{u}-u=2(\sqrt{a b}+\sqrt{(a+1)(b-1)}),
$$

by $(3.20)$,

and

$$
\sqrt{a b}=\sqrt{\frac{\left(1-t^{6}\right)^{2}}{16}}
$$

Equating these identities, we deduce that

$$
\sqrt{(a+1)(b-1)}=\sqrt{\frac{t^{12}-18 t^{6}+1}{16}} .
$$

$$
a=\left(\frac{\sqrt{5}-1}{2}\right)^{3} \frac{1-t^{6}}{4} \text { and } b=\left(\frac{1+\sqrt{5}}{2}\right)^{3} \frac{1-t^{6}}{4} .
$$

We complete the proof by substituting these values into (3.21).

Alternative proof of Theorem 3.2. Recall that, if $k \leq \sqrt{5}-2$, then

$$
\frac{k}{1-k^{2}}\left(\frac{1+k-k^{2}}{1-4 k-k^{2}}\right)^{5}=q(-q ; q)_{\infty}^{24}
$$

and

$$
\left(\frac{k}{1-k^{2}}\right)^{5} \frac{1+k-k^{2}}{1-4 k-k^{2}}=q^{5}\left(-q^{5} ; q^{5}\right)_{\infty}^{24}
$$

The identities (3.22) and (3.23) are found in Ramanujan's lost notebook [18, p. 53], and the first proofs are given in [9, Theorem 4.5].

Divide (3.23) by (3.22) to deduce that

$$
\left(\frac{k\left(1-4 k-k^{2}\right)}{\left(1-k^{2}\right)\left(1+k-k^{2}\right)}\right)^{4}=\left(q^{1 / 6} \frac{\left(-q^{5} ; q^{5}\right)_{\infty}}{(-q ; q)_{\infty}}\right)^{24} .
$$

Taking fourth roots of both sides yields (3.14). The rest of the proof is the same as above. 
4. Evaluations of $R(q)$. Let $q=\exp (-\pi \sqrt{n})$ for a positive rational number $n$ in the sequel.

In the following two corollaries, the explicit formulas for $R(q)$ discussed in Section 3 are used for illustration. However, it should be mentioned that it is much easier to use Theorems 2.1 and 2.3 for computing $R(q)$ than the explicit formulas.

We first reclaim (1.3) and (1.4) in the corollary to Theorem 3.1 below.

COROLlary 4.1. If $R(q)$ and $S(q)$ are defined by (1.1) and (1.2), respectively, then

$$
R\left(e^{-2 \pi}\right)=\sqrt{\frac{5+\sqrt{5}}{2}}-\frac{\sqrt{5}+1}{2}
$$

and

$$
S\left(e^{-\pi}\right)=\sqrt{\frac{5-\sqrt{5}}{2}}-\frac{\sqrt{5}-1}{2} .
$$

Proof. Let $q=\exp (-\pi)$. Since (see [17])

$$
G_{n}=G_{1 / n},
$$

we find from (2.15) that $t_{2}\left(-e^{-\pi}\right)=-1$. Using this value in Theorem 3.1(ii), (i), we deduce (i) and (ii) above, respectively, after lengthy calculations.

We next turn to applications of Theorem 3.2.

Corollary 4.2 .

$$
S\left(e^{-\pi \sqrt{3 / 5}}\right)=\left(\frac{-(3+5 \sqrt{5})+\sqrt{30(5+\sqrt{5})}}{4}\right)^{1 / 5} .
$$

REMARK. This evaluation is mentioned in the incomplete table of $S(q)$ on page 210 in Ramanujan's lost notebook, but the actual value was first introduced and proved by Ramanathan in [13].

Proof (of Corollary 4.2). Let $q=e^{-\pi \sqrt{3 / 5}}$. Then by (2.14),

$$
t_{1}^{6}\left(-e^{-\pi \sqrt{3 / 5}}\right)=-\left(\frac{G_{3 / 5}}{G_{15}}\right)^{6} .
$$

From Weber's table [22, p. 721], or [4, Chapter 34], we find that

$$
G_{15}=2^{-1 / 12}(1+\sqrt{5})^{1 / 3} \text {. }
$$

Using a modular equation of degree 5 [2, p. 282, Entry 13(xiv)], and (1.14), we deduce that

$$
G_{3 / 5}=2^{-1 / 12}(-1+\sqrt{5})^{1 / 3} .
$$


Hence

$$
t_{1}^{6}=-\frac{3-\sqrt{5}}{3+\sqrt{5}}
$$

Substitute (4.2) into (ii) and (iii) to find $k$ and $(1-k) /(1+k)$, then complete the proof using the first equality in (i). However, these calculations are very lengthy.

REMARK. By utilizing (4.2) in the second equality in (i), we can also evaluate $R\left(e^{-2 \pi \sqrt{3 / 5}}\right)$.

We complete this section with an application of Theorem 2.1 and Proposition 2.2.

Corollary 4.3 ([18], p. 210).

$$
S^{5}\left(e^{-\pi / \sqrt{5}}\right)=\sqrt{\left(\frac{5 \sqrt{5}-11}{2}\right)^{2}+1}-\frac{5 \sqrt{5}-11}{2} .
$$

Proof. Let $q=e^{-\pi / \sqrt{5}}$. Then by (2.14) and (4.1),

$$
t_{1}^{6}\left(-e^{-\pi / \sqrt{5}}\right)=-\left(\frac{G_{1 / 5}}{G_{5}}\right)^{6}=-1 .
$$

Recalling that $P_{1}$ is defined in (2.4), from Theorem 2.1(i), (iv), we have

$$
P_{1}^{6}(-q)=\frac{f^{6}(q)}{-q f^{6}\left(q^{5}\right)}=-5 \sqrt{5} .
$$

We complete the proof upon substituting (4.3) into Proposition 2.2(ii).

5. Evaluations of theta-functions. Recall that [2, p. 103]

$$
\varphi\left(e^{-\pi}\right)=\frac{\pi^{1 / 4}}{\Gamma(3 / 4)} .
$$

We can evaluate quotients of theta-functions in the forms of those in Theorems 2.1 and 2.3 as we can determine the Rogers-Ramanujan continued fraction, but by using (5.1), $\varphi\left(e^{-\pi \sqrt{n}}\right)$ and $\psi\left(-e^{-\pi \sqrt{n}}\right)$ may be determined explicitly.

Corollary 5.1. Recall that $\varphi(q)$ and $\psi(q)$ are defined by (1.9) and (1.10), respectively. Then

$$
\begin{gathered}
\frac{\varphi\left(e^{-\pi}\right)}{\varphi\left(e^{-5 \pi}\right)}=\sqrt{5 \sqrt{5}-10} \\
\frac{\psi\left(-e^{-\pi}\right)}{e^{-\pi / 2} \psi\left(-e^{-5 \pi}\right)}=\sqrt{5 \sqrt{5}+10}
\end{gathered}
$$


REMARK. Corollary 5.1(i) is recorded on page 285 in Ramanujan's first notebook. One of the proofs of this evaluation is given by Berndt and Chan [5]. Corollary 5.1(ii) appears to be new.

Pro of (of Corollary 5.1). Let $q=e^{-\pi}$. From Ramanujan's paper [16], [17], Weber's table [22, p. 722], or [4, Chapter 34],

$$
G_{1}=1 \quad \text { and } \quad G_{25}=(1+\sqrt{5}) / 2 .
$$

Thus by (2.14),

$$
t_{1}^{6}\left(-e^{-\pi}\right)=-\left(\frac{G_{1}}{G_{25}}\right)^{6}=-\left(\frac{\sqrt{5}-1}{2}\right)^{6} .
$$

Hence, from Theorem 2.1(iv),

$$
\left(\frac{\varphi\left(e^{-\pi}\right)}{\varphi\left(e^{-5 \pi}\right)}\right)^{2}=-4+2 \sqrt{5}+\sqrt{81-36 \sqrt{5}}=5 \sqrt{5}-10 .
$$

Corollary 5.1(i) follows immediately from (5.4). Applying (5.3) and (5.4) to Theorem 2.1(iii) we deduce Corollary 5.1(ii).

From the theta-function transformation for $\varphi(q)$ [2, p. 102, Corollary] or Theorem 2.3 with $t_{2}=-1$, we find that

$$
\frac{\varphi\left(e^{-\pi / 5}\right)}{\varphi\left(e^{-5 \pi}\right)}=\sqrt{5}
$$

Similarly,

$$
\frac{\psi\left(-e^{-\pi / 5}\right)}{e^{-3 \pi / 5} \psi\left(-e^{-5 \pi}\right)}=\sqrt{5}
$$

Combining these results with Corollary 5.1, we obtain

Corollary 5.2.

$$
\begin{gathered}
\frac{\varphi\left(e^{-\pi / 5}\right)}{\varphi\left(e^{-\pi}\right)}=\sqrt{2+\sqrt{5}} \\
\frac{\psi\left(-e^{-\pi / 5}\right)}{e^{-\pi / 10} \psi\left(-e^{-\pi}\right)}=\frac{1}{\sqrt{2+\sqrt{5}}} .
\end{gathered}
$$

6. Tables of $R(q)$ and $S(q)$. Prior to providing the tables of all the values of $R(q)$ and $S(q)$ found in the literature, we first recall the reciprocity theorems for the Rogers-Ramanujan continued fraction stated by Ramanujan in his second letter to Hardy [16] and his notebooks [15]. If both $\alpha$ and $\beta$ are positive and $\alpha \beta=1$, then 


$$
\begin{aligned}
& \left\{\frac{\sqrt{5}+1}{2}+R\left(e^{-2 \pi \alpha}\right)\right\}\left\{\frac{\sqrt{5}+1}{2}+R\left(e^{-2 \pi \beta}\right)\right\}=\frac{5+\sqrt{5}}{2}, \\
& \left\{\frac{\sqrt{5}-1}{2}+S\left(e^{-\pi \alpha}\right)\right\}\left\{\frac{\sqrt{5}-1}{2}+S\left(e^{-\pi \beta}\right)\right\}=\frac{5-\sqrt{5}}{2} .
\end{aligned}
$$

Ramanathan [10] established analogues of (6.1) and (6.2); namely, if $\alpha$ and $\beta$ are positive and $\alpha \beta=1 / 5$, then

$$
\begin{aligned}
\left\{\left(\frac{\sqrt{5}+1}{2}\right)^{5}+R^{5}\left(e^{-2 \pi \alpha}\right)\right\}\left\{\left(\frac{\sqrt{5}+1}{2}\right)^{5}\right. & \left.+R^{5}\left(e^{-2 \pi \beta}\right)\right\} \\
& =5 \sqrt{5}\left(\frac{\sqrt{5}+1}{2}\right)^{5}
\end{aligned}
$$

$$
\begin{aligned}
\left\{\left(\frac{\sqrt{5}-1}{2}\right)^{5}+S^{5}\left(e^{-\pi \alpha}\right)\right\}\left\{\left(\frac{\sqrt{5}-1}{2}\right)^{5}+\right. & \left.S^{5}\left(e^{-\pi \beta}\right)\right\} \\
& =5 \sqrt{5}\left(\frac{\sqrt{5}-1}{2}\right)^{5} .
\end{aligned}
$$

We see that, from each of (6.1)-(6.4), if we find a particular value of $R(q)$ or $S(q)$, then we easily can find the other. For this reason, we list just one of the two values in each case in the table below.

$$
\begin{gathered}
\text { TABLe of } R\left(e^{-\pi \sqrt{n}}\right) \\
R\left(e^{-2 \pi}\right)=\sqrt{\frac{5+\sqrt{5}}{2}}-\frac{\sqrt{5}+1}{2} \\
R\left(e^{-2 \pi \sqrt{2}}\right)=\frac{\sqrt{5(g+1)+2 g \sqrt{5}}-\sqrt{5 g}-1}{2}, \quad \text { where } \\
\left(g^{3}-g^{2}\right) /(g+1)=(\sqrt{5}+1) / 2
\end{gathered}
$$$$
R\left(e^{-2 \pi \sqrt{5}}\right)=\frac{\sqrt{5}}{1+\left(5^{3 / 4}\left(\frac{\sqrt{5}-1}{2}\right)^{5 / 2}-1\right)^{1 / 5}}-\frac{\sqrt{5}+1}{2}
$$$$
\text { Refs: [10], [12], [16], [21] }
$$$$
R\left(e^{-2 \pi \sqrt{2 / 5}}\right)=-18-5 \sqrt{5}+\sqrt{90(5+2 \sqrt{5})}
$$

Refs: [11], [13]$$
R\left(e^{-2 \pi \sqrt{17 / 5}}\right)=\frac{-111-5 \sqrt{5}+\sqrt{10(2245+999 \sqrt{5})}}{2}
$$

Ref: [10]

Ref: [13] 


$$
R\left(e^{-4 \pi}\right)=\sqrt{c^{2}+1}-c, \quad \text { where } \quad 2 c=1+\frac{5^{1 / 4}+1}{5^{1 / 4}-1} \sqrt{5}
$$

Refs: [4], [6], [11], [15]

$$
R\left(e^{-6 \pi}\right)=\sqrt{c^{2}+1}-c, \quad \text { where } \quad 2 c=1+\frac{60^{1 / 4}+2-\sqrt{3}+\sqrt{5}}{60^{1 / 4}-2+\sqrt{3}-\sqrt{5}} \sqrt{5}
$$

Refs: [4], [6], [7], [15]

$$
R\left(e^{-8 \pi}\right)=\sqrt{c^{2}+1}-c, \quad \text { where } \quad 2 c=1+\frac{3+\sqrt{2}-\sqrt{5}+20^{1 / 4}}{3+\sqrt{2}-\sqrt{5}-20^{1 / 4}} \sqrt{5}
$$

$$
\begin{gathered}
R\left(e^{-16 \pi}\right)=\sqrt{c^{2}+1}-c, \quad \text { where } \\
2 c=1+\frac{5^{1 / 4}(4-\sqrt{2})+1+\sqrt{2}+\sqrt{5}-2^{1 / 4}(3-\sqrt{2}+\sqrt{5}-\sqrt{10})}{5^{1 / 4}(4-\sqrt{2})-1-\sqrt{2}-\sqrt{5}+2^{1 / 4}(3-\sqrt{2}+\sqrt{5}-\sqrt{10})} \sqrt{5}
\end{gathered}
$$

$$
\begin{aligned}
& \text { TABLe of } S\left(e^{-\pi \sqrt{n}}\right) \\
& S\left(e^{-\pi}\right)=\sqrt{\frac{5-\sqrt{5}}{2}}-\frac{\sqrt{5}-1}{2} \\
& \text { Refs: [10], [11], [12], [16], [21] } \\
& S\left(e^{-\pi \sqrt{3}}\right)=\frac{-3-\sqrt{5}+\sqrt{6(5+\sqrt{5})}}{4} \\
& \text { Refs: [8], [14], [18] } \\
& S\left(e^{-\pi \sqrt{5}}\right)=\frac{\sqrt{5}}{1+\left(5^{3 / 4}\left(\frac{\sqrt{5}+1}{2}\right)^{5 / 2}-1\right)^{1 / 5}}-\frac{\sqrt{5}-1}{2} \\
& \text { Refs: [10], [12] } \\
& S\left(e^{-3 \pi}\right)=\sqrt{c^{2}+1}-c, \quad \text { where } \quad 2 c=\frac{(60)^{1 / 4}+2+\sqrt{3}-\sqrt{5}}{(60)^{1 / 4}-2-\sqrt{3}+\sqrt{5}} \sqrt{5}-1 \\
& \text { Ref: [7] } \\
& S^{5}\left(e^{-\pi / \sqrt{5}}\right)=\sqrt{\left(\frac{5 \sqrt{5}-11}{2}\right)^{2}+1}-\frac{5 \sqrt{5}-11}{2} \\
& S^{5}\left(e^{-\pi \sqrt{3 / 5}}\right)=\frac{-5 \sqrt{5}-3+\sqrt{30(5+\sqrt{5})}}{4}
\end{aligned}
$$




$$
\begin{aligned}
& S^{5}\left(e^{-\pi \sqrt{7 / 5}}\right)=-5 \sqrt{5}-7+\sqrt{35(5+2 \sqrt{5})} \\
& \text { Refs: [6], [11], [12], [13], [18] } \\
& S^{5}\left(e^{-\pi \sqrt{9 / 5}}\right)=\sqrt{c^{2}+1}-c, \quad \text { where } \quad 2 c=\frac{2 \sqrt{15}+3 \sqrt{5}-1}{2 \sqrt{15}-3 \sqrt{5}+1} 5 \sqrt{5}-11
\end{aligned}
$$

$$
\begin{gathered}
S^{5}\left(e^{-\pi \sqrt{11 / 5}}\right)=\sqrt{c^{2}+1}-c, \quad \text { where } \\
2 c=\left(\frac{\sqrt{3 \sqrt{5}+7}-\sqrt{3 \sqrt{5}-1}}{\sqrt{9 \sqrt{5}+27}-\sqrt{9 \sqrt{5}+19}} \sqrt{5}\right)^{3}-11
\end{gathered}
$$

$$
\begin{gathered}
S^{5}\left(e^{-\pi \sqrt{13 / 5}}\right)=\sqrt{c^{2}+1}-c, \quad \text { where } \\
2 c=\left(\frac{\sqrt{\sqrt{65}+7}-\sqrt{\sqrt{65}-1}}{\sqrt{\sqrt{65}+9}-\sqrt{\sqrt{65}+7}} \cdot \frac{\sqrt{5}}{2}\right)^{3}-11
\end{gathered}
$$

Ref: [7]

$$
S^{5}\left(e^{-\pi \sqrt{23 / 5}}\right)=-207-95 \sqrt{5}+3 \sqrt{5(1955+874 \sqrt{5})}
$$

Refs: [11], [13]

$$
\begin{gathered}
S^{5}\left(e^{-\pi \sqrt{29 / 5}}\right)=\sqrt{c^{2}+1}-c, \quad \text { where } \\
2 c=\left(\frac{\sqrt{\sqrt{145}+17}-\sqrt{\sqrt{145}+9}}{(\sqrt{\sqrt{29}+2 \sqrt{5}+1}-\sqrt{\sqrt{29}+2 \sqrt{5}-1})^{2}} \sqrt{\frac{5}{2}}\right)^{3}-11
\end{gathered}
$$

Ref: [7]

$$
\begin{gathered}
S^{5}\left(e^{-\pi \sqrt{39 / 5}}\right)=\sqrt{c^{2}+1}-c, \quad \text { where } \\
2 c=\left(\sqrt{5}(\sqrt{65}+8)^{1 / 3}\left(\frac{\sqrt{13}+3}{2}\right)\right)^{3}-11 \\
2 c=\left(\frac{\sqrt{3 \sqrt{41}+25}-\sqrt{3 \sqrt{41}+17}}{(\sqrt{3 \sqrt{41}+23}-\sqrt{3 \sqrt{41}+19})^{2}} \sqrt{10}\right)^{3}-11 \\
2 c=\left(\frac{(\sqrt{\sqrt{265}+16}-\sqrt{\sqrt{265}+12}) 2 \sqrt{5}}{(\sqrt{5 \sqrt{53}+17 \sqrt{5}+2}-\sqrt{5 \sqrt{53}+17 \sqrt{5}-2})^{2}}\right)^{3}-11
\end{gathered}
$$

Ref: [7]

Ref: [7] 


$$
\begin{aligned}
& S^{5}\left(e^{-\pi \sqrt{89 / 5}}\right)=\sqrt{c^{2}+1}-c, \quad \text { where } \\
& 2 c=\left(\frac{\sqrt{9 \sqrt{89}+85}-\sqrt{9 \sqrt{89}+77}}{(\sqrt{9 \sqrt{89}+85}-\sqrt{9 \sqrt{89}+83})^{2}} \sqrt{\frac{5}{2}}\right)^{3}-11 \\
& S^{5}\left(e^{-\pi \sqrt{101 / 5}}\right)=\sqrt{c^{2}+1}-c, \quad \text { where } \\
& 2 c=\left(\frac{\sqrt{5 \sqrt{505}+113}-\sqrt{5 \sqrt{505}+105}}{(\sqrt{13 \sqrt{101}+58 \sqrt{5}+1}-\sqrt{13 \sqrt{101}+58 \sqrt{5}-1})^{2}} \sqrt{\frac{5}{2}}\right)^{3}-11
\end{aligned}
$$

Ref: $[7]$

Acknowledgments. The author is much indebted to Professor Heng Huat Chan for his fruitful suggestions on the proofs of theorems in Section 3. She also thanks her thesis advisor Professor Bruce C. Berndt for his useful comments and encouragement during the writing of this paper.

\section{References}

[1] G. E. Andrews, B. C. Berndt, L. Jacobsen and R. L. Lamphere, The Continued Fractions Found in the Unorganized Portions of Ramanujan's Notebooks, Mem. Amer. Math. Soc. 477 (1992).

[2] B. C. Berndt, Ramanujan's Notebooks, Part III, Springer, New York, 1991.

[3] -, Ramanujan's Notebooks, Part IV, Springer, New York, 1994.

[4] -, Ramanujan's Notebooks, Part V, Springer, New York, 1997.

[5] B. C. Berndt and H. H. Chan, Ramanujan's explicit values for the classical thetafunction, Mathematika 42 (1995), 278-294.

[6] - - - Some values for the Rogers-Ramanujan continued fraction, Canad. J. Math. 47 (1995), 897-914.

[7] B. C. Berndt, H. H. Chan and L.-C. Zhang, Explicit evaluations of the RogersRamanujan continued fraction, J. Reine Angew. Math. 480 (1996), 141-159.

[8] H. H. Chan, On Ramanujan's cubic continued fraction, Acta Arith. 73 (1995), 343-355.

[9] S.-Y. Kang, Some theorems on the Rogers-Ramanujan continued fraction and associated theta functions in Ramanujan's lost notebook, Ramanujan J. 3 (1999), 91-111.

[10] K. G. Ramanathan, On Ramanujan's continued fraction, Acta Arith. 43 (1984), 209-226.

[11] - On the Rogers-Ramanujan continued fraction, Proc. Indian Acad. Sci. Math. Sci. 93 (1984), 67-77.

[12] —, Ramanujan's continued fraction, Indian J. Pure Appl. Math. 16 (1985), 695-724.

[13] - Some applications of Kronecker's limit formula, J. Indian Math. Soc. 52 (1987), 71-89.

[14] - On some theorems stated by Ramanujan, in: Number Theory and Related Topics, Tata Inst. Fund. Res. Stud. Math. 12, Oxford Univ. Press, Bombay, 1989, 151-160. 
[15] S. Ramanujan, Notebooks (2 volumes), Tata Inst. Fund. Res., Bombay, 1957.

[16] —, Collected Papers, Chelsea, New York, 1962.

[17] -, Modular equations and approximations to $\pi$, Quart. J. Math. (Oxford) 45 (1914), 350-372.

[18] - , The Lost Notebook and Other Unpublished Papers, Narosa, New Delhi, 1988.

[19] L. J. Rogers, Second memoir on the expansion of certain infinite products, Proc. London Math. Soc. 25 (1894), 318-343.

[20] G. N. Watson, Theorems stated by Ramanujan (VII): Theorems on continued fractions, J. London Math. Soc. 4 (1929), 39-48.

[21] - Theorems stated by Ramanujan (IX): Two continued fractions, J. London Math. Soc. 4 (1929), 231-237.

[22] H. Weber, Lehrbuch der Algebra, dritter Band, Chelsea, New York, 1961.

Department of Mathematics

University of Illinois at Urbana-Champaign

1409 W. Green Street

Urbana, Illinois 61801

U.S.A.

E-mail: kang@math.uiuc.edu 